\title{
Identifying Awareness Requirements in Face-to-Face Collaborative Applications for Users with Autism Spectrum Disorders
}

\author{
Greis F. Mireya Silva, Alberto Barbosa Raposo
}

\author{
Department of Informatics \\ Pontifical Catholic University of Rio de Janeiro (PUC-Rio) - RJ - Brazil \\ \{gcalpa, abraposo\} @inf.puc-rio.br
}

\begin{abstract}
Individuals with Autism Spectrum Disorders (ASD), mainly those with high severity, have difficulties to identify and understand the others' actions and emotions and, therefore, to understand the basic concepts of a collaborative work, even in face-to-face situations. We developed and evaluated a multitouch collaborative game with youths with high severity in ASD. Despite the positive results to encourage the social interaction, we observed that they had difficulties to understand tasks, to identify their partners, and the workspace. Based on these difficulties, we identify a set of awareness requirements to contribute to the design of awareness mechanisms adequate to face-to-face collaborative applications for individuals with ASD.
\end{abstract}

\section{Introduction}

The development of a collaborative application usually considers that users possess the intrinsic characteristics required to easily perform collaboration activities, involving its three dimensions: communication, cooperation and coordination [Ellis, Gibbs and Rein 1991]. The systems attempt to offer awareness support mechanisms so that the collaborative process is performed effectively and without hiccups. Within CSCW context, "awareness" is defined by Dourish and Bellotti (1992) as: "An understanding of the activities of others, which provides a context for your own activity. This context is used to ensure that individual contributions are relevant to the group's activity as a whole, and to evaluate individual actions with respect to group goals and progress".

A great challenge arises when it comes to design collaborative applications for individuals that present difficulties to understand the most basic concepts of a collaborative activity, including difficulty to interpret what the other person is doing or feeling, even in face-to-face situations. This is the case of individuals with ASD, who also present communication and social interaction deficits, together with restricted and repetitive behavior patterns [APA 2013, WHO 1992]. To strengthen these compromised abilities, it is very important to engage these individuals in collaborative situations, using the appropriate methods to motivate them to identify, learn and develop the activities that encompass a collaborative process. However, current collaborative systems are not designed to take into account these limitations, therefore restricting their use by these individuals.

Studies about face-to-face multitouch collaborative applications for individuals with ASD, mainly for those with low impairment in ASD [Chen 2012, Millen et al. 
2011] have used different strategies [Hourcade et al. 2012, Stone et al. 2011] or restrictions regarding the user interface elements, to motivate/force collaboration among users [Battocchi et al. 2009, Bauminger et al. 2007, Gal et al. 2009, Giusti et al. 2011, Piper et al. 2006], presenting positive results by stimulating users' social interaction abilities, and the potential benefits of the technology to encourage users' gestural and verbal communication. However, even the collaborative applications developed for this public, still do not consider notions of awareness for these users that do not know how to collaborate in a typical sense. In this context, there are still many unanswered questions, especially for people with high impairment in $\mathrm{ASD}^{1}$. For example, what are the needs of individuals with ASD within the context of collaborative work? How can we offer software that allows them to perceive tasks, actions of their peers, their own actions and the workspace? What software characteristics have to be taken into account by developers to offer learning opportunities, regarding the collaboration dimensions, for these users that do not know how to collaborate in a typical sense?

As an initial contribution to this great challenge, we have developed and evaluated a collaborative game for the interaction of two users in a multitouch tabletop, specifically developed for individuals with high severity in ASD. This game includes a set of restrictions called Collaboration Patterns. These patterns restrict, in different ways, user interaction with the interface elements, where they can share resources, exchange information and simultaneously perform activities.

In previous studies [Silva et al. 2014, 2015], we evaluated the benefits of the game in the users with ASD, and we obtained encouraging results, particularly in the user's interest in the game and in the generation of social interaction expressions. However, we observed that users have some difficulties to easily understand the collaborative activities. Therefore, our objective in this work is to identify these difficulties in order to obtain a set of requirements to guide the design of collaborative applications for individuals with ASD.

Our evaluation, in this paper, has focused on the difficulties faced by users during their interaction playing the game, especially those related to the awareness of tasks, game peers, and the context of the group as a whole. Through our analysis of such difficulties, we have verified that it is necessary to not only offer restrictions to motivate collaboration, but also opportunities for collaboration learning. These learning opportunities may be offered by including in the application some awareness mechanisms that are appropriate for users to comprehend the dimensions that involve collaborative work. The results of this evaluation have led to an awareness requirements specification aiming to guide the development of awareness mechanisms adequate to face-to-face collaborative applications designed for individuals with ASD.

In the next section, we present related works about strategies and recommendations for the design of collaborative applications in multitouch tabletops for users with ASD. Then, we present the collaborative game and the collaboration patterns

\footnotetext{
${ }^{1}$ Individuals with severe or marked deficits in verbal and nonverbal social communication skills cause severe impairments in functioning, very limited or limited initiation of social interactions, and minimal response to social overtures from others (according to the severity levels of the Diagnostic and Statistical Manual of Mental Disorders-DSM-5) [APA, 2013].
} 
included in the game. Next, we detail the game evaluation process, performed with users with ASD, the obtained results, and the set of awareness requirements identified in the evaluation process. Finally, we present conclusions and future work.

\section{Related work}

Aiming to stimulate collaboration among individuals with ASD, various studies about collaborative multitouch applications have recommended the inclusion of restrictions in the user interface elements. This type of restriction indirectly induces the users to collaborate [Hornecker 2005], because they offer implicit suggestions to act in predetermined ways or to adopt interaction patterns that indirectly "force" collaboration among users.

Among such studies is the game SIDES [Piper et al. 2006], which requires the collaboration of four users, in its interaction via enforced turn-taking, to accomplish the objective of the game. Giusti and collaborators (2011) propose a set of four restrictions called collaborative patterns, one of them being, for example, choosing together pattern, which requires the touch of two or more users to select one particular object. The authors concluded that the users learned the importance of collaboration as they advanced in the game phases, adequately responding to the collaboration strategies implemented [Weiss et al. 2011].

Similarly, other authors [Battocchi et al. 2009, Bauminger et al. 2007, Gal et al. 2009] have developed two games based on the Enforced Collaboration Paradigm, which consists in forcing the simultaneous interaction of two or more users on the same interface element to move it. Using this paradigm, the Collaborative Puzzle Game [Battocchi et al. 2009] requires that two users must move together each piece of a puzzle to assemble it; and in the StoryTable [Bauminger et al. 2007, Gal et al. 2009] users must create together a common story.

Even studies about applications for individuals with typical development consider that it is important to identify what has to be restricted in a collaborative interface to help people interact problem free. For example, Yuill and Rogers (2012) suggest it is necessary to consider restricting the following aspects: the degree of awareness of user actions and intentions, the degree of control over the interface, and the degree of availability of relevant information.

From the above mentioned related work, we may conclude that interaction restrictions are important to motivate the collaboration among individuals with ASD. The Collaboration Patterns used in the game presented in this work also include a gradual sequence of restrictions to instruct the users on the most basic aspects of collaboration.

Other studies have indicated important characteristics to be considered in the design of collaborative applications for individuals with ASD, which we classify as follows:

Fun applications. Parents of children and adults with ASD have indicated, in an interview performed by Putnam e Chong (2008), the importance of applications to be fun and include learning experiences, in addition to consider each users' specific characteristics and sensorial integration aspects. 
Simple and predictable applications. Understanding simplicity in the sense of "removing unnecessary information and features so that technology is simpler to understand and intuitive to use" [Keay-Bright and Howarth 2012]. Simplicity of user interface elements is mandatory, as the intent is to help users to deal with the complexities of the real work. If users are able to feel comfortable from the beginning in their interaction with the application, then it is possible to encourage them to perform more challenging tasks [Hourcade et al. 2012].

Error agnostic applications. Use of applications with no wrong answers neither error messages [Hourcade et al. 2012, Keay-Bright and Howarth 2012], allowing users not to get frustrated and keep motivated throughout the necessary interaction attempts. Keay-Bright and Howarth (2012) also suggest the exclusion of correct answers and instructions, but ASD therapists suggest that this is very important to encourage work progress, so that users perceive that they are in the right path and get even more motivated to continue interaction with the system.

Representation of people in the real world. Such representation may be done through caricatures or photographs, according to the specific characteristics of the users, as some individuals with ASD may see caricatures/avatars as an easier to understand element than common gestural expressions [Moore et al. 2005] and for recognition of emotions [Rosset et al. 2008]. Other individuals may find it easier to identify individuals through photographs or more realistic faces, as photographs may be intuitively more useful when the user is going to interact with the real world [Moore et al. 2005].

Additionally, Zancanaro and collaborators (2010) state that one should notice the following aspects to support the acquisition of social competence by individuals with ASD: consider the specific interests of users and their strong and weak abilities to generate motivation to perform the task; consider up to which point each user is capable of cooperating with others and has perception of them; offer content variety and a feedback with auditory and visual characteristics, according to the user actions level; offer from very simple synchronized actions up to collaborative actions that involve verbal communication or negotiations; and interact accompanied by human meditation.

Moreover, we considered recommendations presented in studies of face-to-face collaborative systems for individuals with typical development, but that we also consider appropriate for individuals with ASD, for its facilitating characteristics. For example, Haller and collaborators (2010) suggest that it is important to provide an environment that contributes to an easy user adaptation and manipulation of interface elements, this way the cognitive work load could be minimized.

However, the recommendations found in the literature are more closely related to interface characteristics to facilitate user interaction, and not specifically related to support awareness. The requirements identified in the present work propose recommendations focused on awareness, which should are also integrated to the gradual sequence of restrictions.

\section{Collaborative Game PAR}

We developed PAR (acronym in Portuguese for Ask, Help and Receive), a multitouch collaborative tabletop game to encourage social interaction among individuals with high 
impairment in ASD. We chose multitouch technology by its advantages on collaborative work. This technology allows high levels of awareness and more fluent interactions [Hornecker et al. 2008].

PAR includes Collaboration Patterns, a set of interaction strategies on elements in a multiuser interface to gradually encourage collaboration among individuals with ASD, mainly those with more level of severity in ASD. Collaboration Patterns were designed based on the constraints proposed by Giusti and collaborators (2011), such as, "Different role pattern", which assigns different roles for each user, and "Constraints on objects pattern", which requires two or more users to move an object. The set includes a specific sequence of four Collaboration Patterns. We proposed this sequence following recommendations of experts in ASD. They indicated the collaboration is a complex area for people with ASD with high impairment; therefore, this must be carefully and gradually encouraged. The following sequence of Collaboration Patterns was used in PAR and indicates a gradual reinforcement of collaborative activities:

Passive Sharing Pattern. Each user has a different role to share resources. The tasks are just action and response from one user to another. To 'share resources', each user has only to realize his/her own task and know the result of the tasks of the partner, independently of identifying who executed the task and how it was executed.

Active Sharing Pattern introduces the importance of recognizing the role of the partner in collaboration. This is achieved by requiring 'information exchange' between users, in addition to sharing resources. Each user receives information from the partner about how to cooperate.

Joint-Performance Pattern uses the same principles as "Active Sharing Pattern" and introduces cooperative 'simultaneous actions' to help the other when needed. Users now realize that the participation of both is strictly necessary to achieve a goal, recognizing that they are involved in a collaborative activity.

Unrestricted Interaction Pattern. This pattern does not assign roles or constraints to users, allowing them to collaborate and develop 'free strategies' for sharing information, cooperating, and helping each other. This pattern is offered to users after they have interacted with each restricted patterns.

PAR game requires collaboration of two users around a multitouch tabletop, in order to get the sport pieces (shirt, shorts and sneakers) and to dress the soccer players of a team (Figure 1). The sports pieces are randomly distributed on three higher shelves of a warehouse. These pieces need to be taken down so the players can wear them. On the side of each shelf, there is a box. This box serves as a container. One user must put one sport piece into a box that serves as a container and send it to the second user. In the lower part of the warehouse, there is a cart. Second user must receive each sport piece in this cart. When the cart is full with the three sport pieces, it is necessary to take it to the parking lot and give the pieces one by one to the player in a row.

PAR game has three collaborative phases. Each user will have a different role according their place around the multitouch tabletop. One user is located at the upper side of the table (User 1), and the second user is located at the lower side of the table (User 2). At the time of dressing each player, users may move to the right side of the table (Figure 1). The first phase includes the Passive Sharing Pattern (Figure 1.a). The 
role of user 1 is to take any sport piece and put it in the box on the shelf. The role of user 2 is to move the cart and take it to the shelf of the descending box to receive the piece sent by User 1. The second phase adds the Active Sharing Pattern (Figure 1.b), because User 1 must have information about the three sport pieces that should be sent. User 2 should ask for any piece by pressing a button. User 1 should then put the piece requested by the partner into the box to send it.
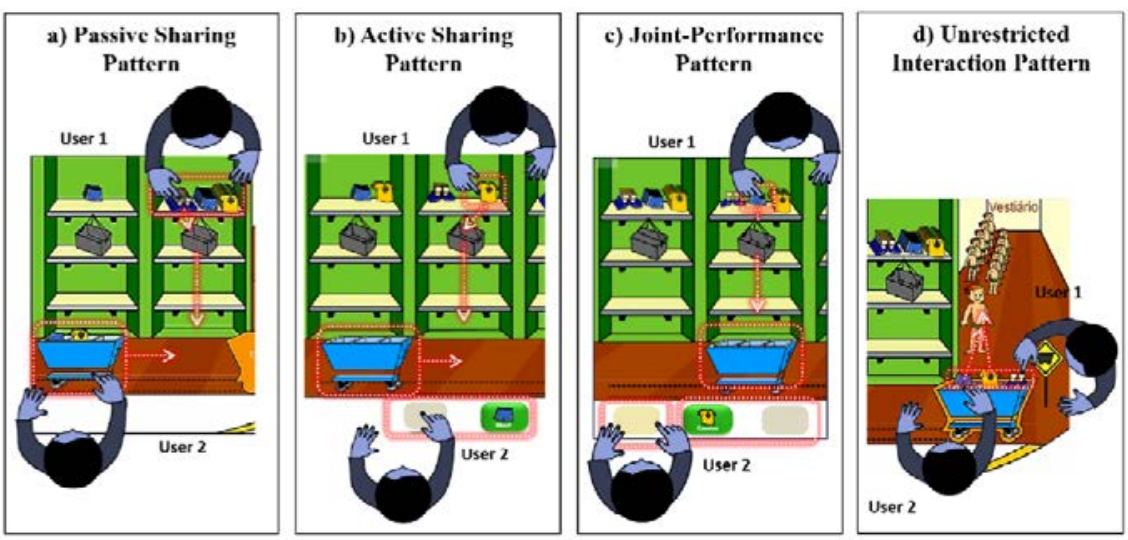

Figure 1. Collaboration Patterns in Collaborative Game PAR.

In addition to the tasks of the previous phases, in the third phase the boxes are already closed at the time that each piece is requested. Therefore, the Joint-Performance Pattern is applied because the box must be opened by both users. User 2 must press a button to open the boxes while User 1 takes and puts the piece requested into the box (Figure 1.c). In the three phases, when the cart is filled with three pieces, User 2 should move the cart to the parking lot. Here, the Unrestricted Interaction Pattern is applied, because each user may take any piece of the car in any order to dress the soccer player (Figure 1.d).

\section{Evaluation}

In this work, the main research questions of the evaluation include: What difficulties users had in the collaborative process during the game? Are these difficulties related with awareness of tasks, partners or workspace? What are the requirements of users according to their interaction in the game?

\subsection{Evaluation Methods}

Participants. We evaluated PAR with five youths with high impairment in ASD with ages between 10 and 17 years. These youths have high level of impairment in social interaction and collaboration skills. These users have difficulties in verbal communication, visual contact, and lack initiative to interact and share with others. Some users have behavioral problems and difficulty in respecting rules in a group. We named each user with letters from A to E: A is a 17-year-old boy, B is a 16-year-old boy, $\mathrm{C}$ is a 15-year-old girl, $\mathrm{D}$ is a 14-year-old boy, and $\mathrm{E}$ is a 10-year-old girl. The ethical questions were considered at all stages of the study. Parents of youths signed the respective Term of Free and Clarified Consent to authorize the study and the evaluation process with the youths. 
Environment. We performed the evaluation in an institution for people with ASD, where we installed the multitouch tabletop and three cameras located at different angles focusing on the table. This arrangement allowed recording both user-system and user-user interactions.

Procedure. We performed 51 evaluation sessions with ten pairs of users. Each session lasted between 5 and 15 minutes. The evaluation routine was conducted so that each user interacted with the game in the order of phases. During the evaluation sessions, users were accompanied by a therapist who was willing to help and motivate them if needed.

Measures. We analyzed in each phase of the game the users' collaborative behavior related to the understanding of the tasks, the partners' awareness, and understanding of past, current and future activities. Due to the difficulties of users with $\mathrm{ASD}$, it is not possible to anticipate their probable actions or to make a previous categorization of actions. Thus, we made a categorization from both the observations and recordings during the evaluation. For the parts of the game that include the collaboration patterns with restrictions, we classified the users' difficulties related to task awareness, social awareness, and workspace awareness, defined below:

Task awareness refers to the understanding of the tasks and of what the group needs to accomplish their realization [Belkadi et al. 2013], involving task information, such as, what are its objectives, structure, and how to compete it.

Social awareness refers to the knowledge available about the other collaborators within the social context [Belkadi et al. 2013], about the social connections within the group, involving who is the group, its objective, structure, participants.

Workspace awareness refers to the updated knowledge about interactions of collaborators within the shared workspace [Gutwin and Greenberg 1996], involving aspects like: where are the participants working, what are they doing, who is responsible, with which interface elements are they interacting, what are they going to do.

\section{Results}

As highlighted in [Silva et al. 2014, 2015], the interest of the users increased with each phase in the game. Collaboration Patterns applied in the game generated in the users greater motivation to perform the tasks and to interact whit their partners through verbal or gestural expressions, such as have physical contact, see, ask for help, rectify, complain, laugh, commemorate, reject, and encourage. However, after the analysis performed in this work, we also highlighted that during the three phases of the game, in the restricted patterns, users also had some difficulties. We categorized these difficulties into three categories, as detailed in Table 1.

In the first phase of the game, with the Passive Sharing Pattern, users had the larger number of difficulties in the three awareness dimensions. In the second and third phases, the number of difficulties was decreasing in the social and workspace awareness (Figure 2). Users had more difficulties in task awareness with the joint-performance pattern in the third phase of the game, maybe because this phase demanded greater concentration to perform the tasks in the game. 
Table 1. Categories of users' difficulties during the game, classified by different kinds of awareness.

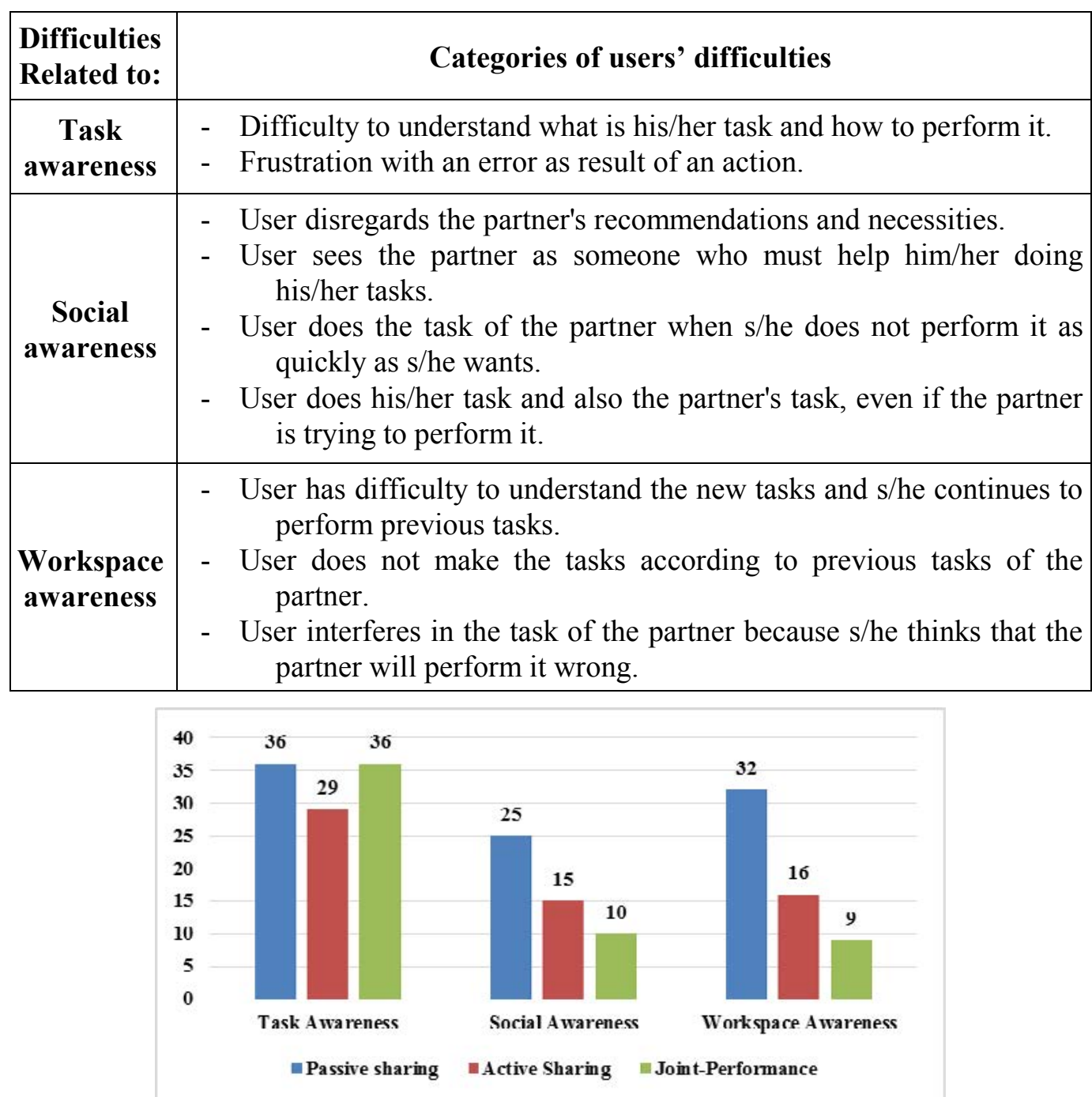

Figure 2. Number of user's difficulties observed in the restricted Collaboration Patterns during all the evaluation sessions.

The number of difficulties related to social awareness is inferior to the number in task and workspace awareness (Figure 2). But that number can be related to the difficulties that some users had even to involve themselves in the collaborative activities. Then, these users perform neither positive nor negative actions related with workspace. Most users (B, C, D, and E) had difficulties related to task awareness, they needed more motivation and orientation to perform the tasks, such as, repeat explanations about the movements required on elements of the game, and indicate who must do the task. Related to social awareness, some users $(\mathrm{B}, \mathrm{E})$ had more difficulty to both identify the other as his/her collaboration partner and understand the importance of wishes and interactions of the partner. This difficulty was reduced throughout the phases of the game. Related to workspace awareness, most users (B, C, D, and E) had sometimes difficulty to respect the workspace of other, interfering in their tasks and interactions. 
In the first phase of the game (Passive Sharing Pattern), user $\mathrm{C}$ had all difficulties categorized in Table 1 at least once. User A had only some difficulties to both follow the wishes of the partner and perform his task according previous tasks of the partner (Figure 3). In the second phase (Active Sharing Pattern), most users had less difficulty (Figure 3) in most of the aspects categorized in Table 1. User E had difficulty to respect the workspace of the partner, but this difficulty decreased in the third phase of the game. Throughout the sequence of Collaboration Patterns, user E learned to respect rules in the collaborative work and waited for the response of the partner. User D had kept a large number of difficulties in the three phases of the game (Figure 3), related to understand what is the task and how to do it. This is the user with most impairment in ASD. He only performed the tasks when the therapist or the partner indicates him, but he does not interest in performing a collaborative work, then it was not possible to observe other difficulties related to social and workspace awareness.

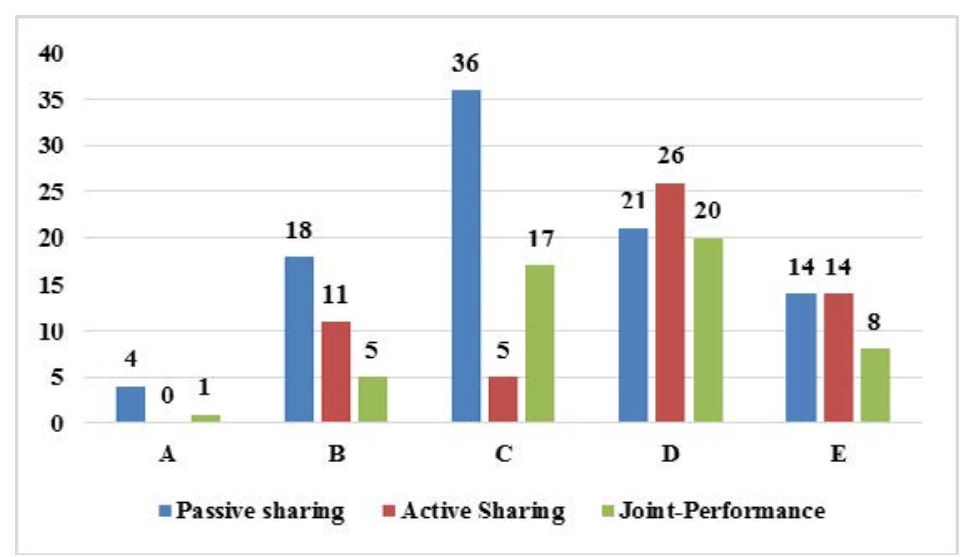

Figure 3. Total number of difficulties of each user in the restricted collaboration patterns during all evaluation sessions.

Since the unrestricted interaction pattern allows a free interaction between users, we did not classify difficulties, but we classified three types of what we called noncollaborative actions, characterized by the disinterest of one or both users (Table 2). These non-collaborative actions were decreasing throughout the phases of the game. In the first phase, four pairs of users performed the three non-collaborative actions while they suit the soccer players. In the second phase, three pairs of users performed only two of these actions. In the third phase, only one pair of users performed once these actions.

Table 2. Types of non-collaborative actions performed by pairs of users during the unrestricted interaction pattern.

\begin{tabular}{|c|c|c|c|}
\hline \multirow{2}{*}{ Non-Collaborative Actions } & \multicolumn{3}{|c|}{ Phases of the game } \\
\hline & 1st & $2 \mathrm{nd}$ & 3th \\
\hline $\begin{array}{l}\text { One user dresses the soccer player and the partner does not show } \\
\text { any interest in collaborating. }\end{array}$ & $\begin{array}{l}\mathrm{BD} \\
\mathrm{CE}\end{array}$ & $\begin{array}{l}\mathrm{BD} \\
\mathrm{DE}\end{array}$ & $\mathrm{CD}$ \\
\hline $\begin{array}{l}\text { Users try to take turns and dress the soccer player, but when one } \\
\text { of them cannot perform the task, the partner becomes aggressive, } \\
\text { discouraging the interaction of the other. }\end{array}$ & $\mathrm{BC}$ & $\mathrm{CD}$ & \\
\hline $\begin{array}{l}\text { Both users need constant motivation to try to dress the soccer } \\
\text { player. }\end{array}$ & $\mathrm{CD}$ & & \\
\hline
\end{tabular}


PAR evaluation results have led to the identification of users' awareness requirements during their interaction with the game. These requirements are classified in: task awareness, social awareness, and workspace awareness, as detailed in the next subsection.

\subsection{Awareness Requirements}

The awareness requirements presented here were obtained according to the difficulties of users in their interaction with PAR game and the empirical evaluation results. We also considered some aspects mentioned in other studies (Section 2) together with experts in $\mathrm{ASD}$, to define these requirements.

We organized the awareness requirements obtained in groups of questions (Table 3) that should be responded by developers to support each type of awareness regarding the interaction of users with ASD with face-to-face collaborative applications. These questions are similar to questions for the specification of a system focused on awareness for individuals with typical development [Belkadi et al. 2013], but they comply with difficulties that are particular of individuals with ASD. They do not include all the possible questions for people with typical development, and the proposed questions aim to involve the more specific needs of users with ASD.

Task awareness requirements. To promote knowledge of the actions being performed, and encourage the users identify themselves as responsible for certain tasks, the collaborative applications shall:

- Explain in detail for each user what is(are) his/her task(s) in the system, and everything related to it(them), offering a highly visual and auditory content, avoiding the use of text.

- Use some means of highlighting the task of each user (greater visibility, colors, lights, images, audios, etc.) more than the other elements of the interface, while the user is interacting with that particular task.

- Explain the importance of performing each task and the objective to be accomplished, through images and/or audios. This explanation may be presented at any time, depending on the difficulty that each user may have to understand it.

- Actively display the user name/photo at the screen, hinting that it is his/her turn to perform the task. Constantly motivate the user to perform the task, always encouraging by his/her name and showing elements at the interface that are of his/her interest.

- Show the performance of the task in parallel to the user interaction, in a way that the user "imitates" the actions shown and required by the system.

- Guide the user in each step of the task, indicating which actions to perform, with which elements and types of interactions.

- Show through animations, and/or audios, the explanation about the result of the task. In case the action has been in error, the system shall not show the wrong answers and/or negative messages, but instead an invitation to try again, with the explanation of the actions that must be done to be successful in the new attempt. 
Table 3. Questions for awareness support in collaborative applications for individuals with ASD

\begin{tabular}{|c|c|}
\hline $\begin{array}{c}\text { Type of } \\
\text { awareness }\end{array}$ & Questions \\
\hline $\begin{array}{l}\text { Task } \\
\text { awareness }\end{array}$ & $\begin{array}{l}\text { - Identification of tasks: What do I know about the task, its structure, } \\
\text { content and context? } \\
\text { - Objectives: What is the objective of the task? Why should I perform } \\
\text { it? } \\
\text { - Execution: How do I identify that I am responsible for the execution } \\
\text { of the task? What steps should I follow to complete the task? } \\
\text { What elements I need to perform on the interface to complete it? } \\
\text { - Evaluation: How task execution will be evaluated? }\end{array}$ \\
\hline $\begin{array}{l}\text { Social } \\
\text { awareness }\end{array}$ & $\begin{array}{l}\text { - Identification of work performed in group: How do I identify that I } \\
\text { belong to a certain group? How and when may I contribute with } \\
\text { my group? } \\
\text { - Objectives: Why should I interact with other to complete my } \\
\text { activity? Why do we work together? } \\
\text { - Identification of partners and their tasks: Who are my partners? } \\
\text { What are my roles in this group? What are the roles of my } \\
\text { partners? How can I identify when my partner(s) need(s) my } \\
\text { collaboration? }\end{array}$ \\
\hline $\begin{array}{l}\text { Workspace } \\
\text { awareness }\end{array}$ & $\begin{array}{l}\text { - Identification of shared workspace: Where am I working? Where are } \\
\text { my partners working? } \\
\text { - Knowledge about partners interaction within the shared workspace: } \\
\text { Who is responsible for an action or object? How can I help my } \\
\text { partners to complete the tasks? } \\
\text { - Identification of what is happening now and is going to happen } \\
\text { within the workspace: What are the current tasks of my partners? } \\
\text { What are they going to do? }\end{array}$ \\
\hline
\end{tabular}

Social awareness requirements. To encourage the users to be conscious of their partners and everyone's interactions, the collaborative applications shall:

- Offer a detailed explanation, using audiovisual means, about who is part of the group, highlighting each user as an essential part to the work to be performed.

- Inform about who finished the performance of some task and who must continue to collaborate with the previous task (calling the user by the name), and also show the respective explanation about how to interact and in which elements of the interface.

- Reserve a space to place the photos and names of each user, so that they feel identified.

- Offer intuitive ways, preferably images and sounds, to explain which are the functions of each member of the group and in which moment they should be performed.

- Offer distinct ways for each participant to call for the attention of the other, such that this participant can quickly perceive that his/her partner is trying to communicate. 
Workspace awareness requirements. To introduce the users to the knowledge of workspace, the collaborative applications shall:

- Reserve space at the interface for each member of the group, identifying it with distinct characteristics for each one, including his/he photo and name.

- Offer a distinct way to highlight the participation of each user and the place around the interface in which he/she is interacting.

- Invite each participant to cooperate with his/her partner in the required moment, encouraging him/her that his/her help is very important to reach the objective.

- Block and free the elements of the interface in the adequate moments to facilitate users' interaction and encourage them to collaborate.

- Show the actions that are being performed at the workspace and the information about who is doing.

We suggest that each type of awareness requirement be included in collaborative applications sequentially, in order to gradually involve the users in each one of the dimensions that comprise the 3C collaboration model [Fuks et al. 2007]. This has the objective to get users to know each aspect of collaboration, and gradually understanding it as a whole. The $3 \mathrm{C}$ collaboration model includes the inter and intra-relationship of three dimensions: communication, which consists of the conversation through the exchange of messages; cooperation, which are interventions performed by participants within the shared workspace; and coordination, which consists in the management of participants, activities and resources [Fuks et al. 2007, 2008].

Initially, we suggest approximating the users to the knowledge about the tasks to be executed in a collaborative environment (task awareness) incorporating cooperation activities. Then, to incorporate some aspects to approximate the knowledge about work partners (social awareness) with communication activities. Finally, to approximate the knowledge about activities performed within the workspace (workspace awareness) incorporating coordination activities.

\section{Conclusions}

Individuals with ASD present several difficulties in communication and social interaction. They present difficulty to understand even basic concepts of a collaborative activity. Studies about collaborative applications for this public proposed different restrictions and recommendations for applications in order to motivate/force the collaboration among users. However, there are few studies on aspects of awareness for these individuals who do not know how collaborate. Our objective in the present work, as an initial contribution for this challenge, was to identify the difficulties faced by youths with ASD through their interaction in a collaborative game for multitouch tabletop, in order to obtain a set of requirements to guide the design of collaborative applications for individual with ASD, mainly to those with high severity.

We evaluated the collaborative game PAR with youths with ASD between 10 and 17 years, diagnosed with higher level of severity. Despite the encouraging results of the game to engaging users in an attractive experience, gradually encouraging social interaction as mentioned in the previous studies [Silva et al. 2014, 2015], we found, in 
this work, that these youths had different difficulties related to the understanding of tasks and how to perform them (task awareness), to the recognition of a partner (social awareness), and to the understanding of the actions and tasks in the workspace (workspace awareness). The results about users' difficulties during their interaction with the game indicated that these users need even more motivation to perform a collaborative work. It is not sufficient to provide easy tasks or restrictive elements to motivate/force the collaboration. It is necessary to offer learning opportunities on how to collaborate, since this area is unknown or difficult for users with ASD. These learning opportunities can be offered by the gradual stimulation of users' awareness through appropriate awareness mechanisms in the interface. It is also important that users gradually understand each dimension of collaboration (cooperation, communication, coordination).

Awareness mechanisms can be developed based on the awareness requirements identified in this work. We believe that these requirements are a promising tool to design appropriate awareness mechanisms to facilitate the users' interaction with the system and with their partners, and to contribute to their learning about a collaborative work. This tool provides a stimulant first step for future research on collaborative systems for individuals with ASD, especially those with high impairment.

As future research, we will develop collaborative applications following these requirements, and then, evaluate the application with users with $\mathrm{ASD}$, in order to identify benefits, limitations, and possible challenges of this approach.

\section{Acknowledgments}

Greis Silva thanks FAPERJ for the support granted to her work (process \#101.541/2014). Alberto Raposo and Greis Silva thanks FAPERJ for supporting this research (Assistive Technology Program, process \#E-26/190.243/2013).

\section{References}

American Psychiatric Association APA. (2013). "Diagnostic and statistical manual of mental disorders, DSM-5®”. American Psychiatric Pub. Retrieved March 15, 2015 from http://goo.gl/aiFD4W

Battocchi, A., Pianesi, F., Tomasini, D., Zancanaro, M., Esposito, G., Venuti, P., Ben Sasson, A., Gal, E., and Weiss, P.L.T. (2009). "Collaborative Puzzle Game: a tabletop interactive game for fostering collaboration in children with Autism Spectrum Disorders (ASD)". In: Proceedings of the ACM International Conference on Interactive Tabletops and Surfaces (ITS '09), 197-204.

Bauminger, N., Goren-Bar D., Gal E., Weiss, P.L.T., Yifat, R., Kupersmitt, J., Pianesi, F., Stock, O., and Zancanaro, M. (2007). "Enhancing Social Communication in HighFunctioning Children with Autism through a CoLocated Interface". In: Multimedia Signal Processing, 2007. MMSP 2007. IEEE 9th Workshop on, 18-21.

Belkadi, F., Bonjour, E., Camargo, M., Troussier, N., and Eynard, B. (2013). "A situation model to support awareness in collaborative design". International Journal of Human-Computer Studies, 71, 1: 110-129. 
Chen Weiqin. (2012). "Multitouch Tabletop Technology for People with Autism Spectrum Disorder: A Review of the Literature". In: Proceedings of the 4th International Conference on Software Development for Enhancing Accessibility and Fighting Info-exclusion (DSAI 2012), 198-207.

Dourish, P., and Bellotti V. (1992). "Awareness and coordination in shared workspaces". In: Proceedings of the 1992 ACM conference on Computer-supported cooperative work (CSCW '92), 107-114.

Ellis, Clarence A., Gibbs, Simon J., and Rein, G. (1991). "Groupware: some issues and experiences.Commun". ACM , 34, 1: 39-58.

Fuks, H., Raposo, A., Gerosa, M., Pimentel, M., and Lucena, C. (2007). "The 3C Collaboration Model". The Encyclopedia of E-Collaboration, Ned Kock (org), 637644.

Fuks, H., Raposo, A., Gerosa, M., Pimentel, M., Filippo, D., and Lucena, C. (2008). "Inter-and intra-relationships between communication coordination and cooperation in the scope of the 3C Collaboration Model". In: Computer Supported Cooperative Work in Design (CSCWD'08), 148-153.

Gal, E., Bauminger, N., Goren-Bar, D., Pianesi, F., Stock, O., Zancanaro, M., and Weiss, P.L.T. (2009). "Enhancing Social Communication in High-Functioning Children with Autism through a Co-Located Interface". AI \& Society, 24, 1: 75-84.

Giusti, L., Zancanaro, M., Gal, E., and Weiss, P.L.T. (2011). "Dimensions of Collaboration on a Tabletop Interface for Children with Autism Spectrum Disorder". In: Proceedings of the SIGCHI Conference on Human Factors in Computing Systems (CHI'11), 3295-3304.

Gutwin, Carl and Greenberg, Saul. (1996). "Workspace Awareness for Groupware”. In: Conference Companion on Human Factors in Computing Systems (CHI '96), 208209. http://doi.acm.org/10.1145/257089.257284

Haller, M., Forlines, C., Koeffel, C., Leitner, J., and Shen, C. (2010). "Tabletop Games: Platforms, Experimental Games and Design Recommendations". Art and Technology of Entertainment Computing and Communication, 271 - 297.

Hornecker, Eva. (2005). "A design theme for tangible interaction: embodied facilitation". In: Proceedings of the ninth conference on European Conference on Computer Supported Cooperative Work (ECSCW'05), 23-43.

Hornecker, Eva, Marshall, P., Dalton, N. S., Rogers, Y. (2008). "Collaboration and Interference: Awareness with Mice or Touch Input". In: Proceedings of the 2008 ACM Conference on Computer supported cooperative work (CSCW'08), 167-176.

Hourcade, J. P., Bullock-Rest, N. E., and Hansen, T. E. (2012). "Multitouch tablet applications and activities to enhance the social skills of children with autism spectrum disorders". Personal and Ubiquitous Computing, 16, 2: 157-168.

Keay-Bright, W., and Howarth, I. (2012). "Is simplicity the key to engagement for children on the autism spectrum?". Personal Ubiquitous Computing. 16, 2: 129-141. 
Millen, L., Hawkins, T., Cobb, S., Zancanaro, M., Glover T., Weiss, P.L.T, and Gal, E. (2011). "Collaborative Technologies for Children with Autism". In: Proceedings of the International Conference on Interaction Design and Children (IDC'11), 246-249.

Moore, D., Cheng, Y., McGrath, P., and Powell, N. J. (2005). "Collaborative virtual environment technology for people with autism". Focus on Autism and Other Developmental Disabilities, 20, 4: 231-243.

Piper, A., O’Brien, E., Morris, M. R., and Winograd, T. (2006). "SIDES: A Cooperative Tabletop Computer Game for Social Skills Development". In: Proceedings of the 20th Anniversary Conference on Computer Supported Cooperative Work (CSCW'06), 1-10.

Putnam, C., and Chong, L. (2008). "Software and technologies designed for people with autism: what do users want?". In: Proceedings of the 10th international ACM SIGACCESS conference on Computers and accessibility (ASSETS '08), 3-10.

Rosset, B. D., Rondan, C., Da Fonseca, D., Santos, A., Assouline, B., and Deruelle, C. (2008). "Typical Emotion Processing for Cartton but not for Real Faces in Children with Autistic Spectrum Disorders". Journal of Autism and Developmental Disorders, 38, 5: 919-925.

Silva, G. F. M., Raposo, A., and Suplino, M. (2014). Par: A collaborative game for multitouch tabletop to support social interaction of users with autism.Procedia Computer Science, 27, 84-93.

Silva, G. F. M., Raposo, A., and Suplino, M. (2015). Exploring collaboration patterns in a multitouch game to encourage social interaction and collaboration among users with autism spectrum disorder. Computer Supported Cooperative Work (CSCW), 24(2-3), 149-175.

Stone, B., Beach, L., Ganci, A., Jones, T., Ribeiro, B., Strouse, E., and Woolley, E. (2011). "Immersive Learning: Developing an Interactive Touch Screen Learning Module for Children with Autism". In: Proceedings of the 13th International Conference on Engineering and Product Design Education (E\&PDE'11), 684-689.

Weiss, P.L.T., Gal, E., Eden, S., Zancanaro, M., and Telch, F. (2011). "Usability of a multi-touch tabletop surface to enhance social competence training for children with Autism Spectrum Disorder". In: Proceedings of the Chais conference on instructional technologies research, 71-78.

World Health Organization. (1992). "The ICD-10 classification of mental and behavioural disorders: clinical descriptions and diagnostic guidelines". Geneva. Retrieved March 15, 2015 from http://goo.gl/kEBJiR

Yuill, Nicola and Rogers, Yvonne. (2012). "Mechanisms for collaboration: A Design and Evaluation Framework for Multi-User Interfaces". ACM Transactions on Computer-Human Interaction (TOCHI), 19, 1: 1- 25.

Zancanaro, M., Gal, E., Parsons, S., Weiss, T., Bauminger, N., and Cobb, S. (2010). "Teaching social competence: in search of design patterns". In: Proceedings of the 9th International Conference on Interaction Design and Children (IDC '10), 270-273. 